\title{
Correction to: Inhalative sedation with small tidal volumes under venovenous ECMO
}

\author{
Axel Rand ${ }^{1}$ (D . Peter K. Zahn ${ }^{1} \cdot$ Thomas A. Schildhauer $^{2} \cdot$ Christian Waydhas $^{2,3} \cdot$ Uwe Hamsen $^{2}$
}

Published online: 5 April 2018

(c) The Japanese Society for Artificial Organs 2018

Correction to: Journal of Artificial Organs

https://doi.org/10.1007/s10047-018-1030-9

In the original publication, the affiliations of fourth and fifth authors were published incorrectly. The corrected affiliations are given in this correction.

The original article can be found online at https://doi.org/10.1007/ s10047-018-1030-9.

Axel Rand

axel.rand@gmail.com

Peter K. Zahn

zahn@ains-bergmannsheil.de

Thomas A. Schildhauer

sabine.slavik@bergmannsheil.de

Christian Waydhas

christian.waydhas@bergmannsheil.de

Uwe Hamsen

uwe.hamsen@bergmannsheil.de

1 BG Universitätsklinikum Bergmannsheil, Klinik für Anästhesiologie, Intensiv-, Palliativ- und Schmerzmedizin, Bochum, Germany

2 BG Universitätsklinikum Bergmannsheil, Klinik und Poliklinik für Chirurgie, Bochum, Germany

3 Universität Duisburg-Essen, Medizinische Fakultät, Essen, Germany 